\title{
A reliability engineering study for a near surface radioactive waste repository using genetic algorithm
}

\author{
L. A. Aguiar ${ }^{1}$ \& V. C. Damaso ${ }^{2}$ \\ ${ }^{I}$ Núcleo de Análise de Sistemas Ambientais - NASA (UFRJ/CNPq), \\ Brazil \\ ${ }^{2}$ Centro Tecnológico do Exército (CTEx), Brazil
}

\begin{abstract}
Infiltration of water into a waste disposal facility and into the waste region is the main factor inducing the release of radionuclides from a disposal facility. Since infiltrating water flow is dependent on the natural percolation at the site and the performance of engineered barriers, its prediction requires modelling of unsaturated water flow through intact or partially/completely failed components of engineered barriers. These include the cover systems, concrete vault, backfill, waste forms, and overpacks. This paper aims to carry out a performance study regarding a near surface repository in terms of reliability engineering. It is assumed that surface water infiltrates through the barriers reaching the matrix where radionuclides are contained, thus releasing them into the environment. The repository consists of a set of barriers which are considered saturated porous medium, except geosphere. A genetic algorithm is employed to establish an optimized configuration to the structure of the repository, by determining the thicknesses of barriers and waste packages arrangement, taking into account the engineering and radiological constraints. Such restrictions aim to keep the risks associated with the migration of radionuclides at a low level.
\end{abstract}

Keywords: radioactive waste repository, reliability engineering, optimization.

\section{Introduction}

Radioactive waste is an inherent result of nuclear energy generation and the use of radioactive material in industry, science, and medicine. Low and intermediate 
level wastes represent more than $90 \%$ of the total volume of radioactive waste, even though they have only a small fraction of total activity produced in the world. This type of waste should be managed to reduce the associated risks to acceptable levels. The deposition system safety is determined by the performance of its individual components, by shape and containment of the wastes, engineering barriers and natural barriers (host environment). Previously, multiple barriers systems were regarded as groups of independent individual barriers that worked in sequence [1,2]. However, this concept is currently seen in a more integrated and synergic way, as complementary barriers that operate simultaneously and together [3].

The repository risk assessment can provide important information in the selection of barriers. The key point in this choice is the scale of time during which the barrier should work, that can be in the order of hundreds of years, according to the International Atomic Energy Agency. The life time of the engineering barriers system used to contain the radioactive waste significantly affects the repository reliability.

Several studies for safety evaluation of radioactive waste disposal have been done since the 1970's. Among other references, the works presented by Bertozzi et al. [4], Pritzker and Gassmann [5], Garrick [6] and Malbrain [7] may be cited for high level radioactive wastes, and the works presented by Kim et al. [8], Han et al. [9], Little and Penfold [10] and Ene [11] for low and intermediate level radioactive wastes. Each work addresses, in a singular manner, all or a part of the safety assessment or performance evaluation, either by deterministic models or probabilistic modelling.

A reliability modelling for packages of high level waste has been developed. Chesnut [12] has dealt with the mean time to failure (MTTF) for a single package and for multiple packages, while Ananda [13] has used the conditional approach for failure of the packages caused by the high temperature of the high level waste. Ananda et al. [14] has extended the conditional approach for multiple packages, more precisely, for two barriers. For packages of low and intermediate level, Aguiar et al. [15] have discussed the reliability for a large number of packages. This discussion is based on the use of probability distribution that best represents the MTTF of the packages. The study's conclusions show the need for using a more complex system, with multiple engineering barriers, necessary for a more reliable retention of radioactive material.

Mathematical models are widely used within the performance assessment of radioactive waste repositories to describe the behaviour of groundwater systems under the various physical conditions encountered throughout the long time scales involved. The effectiveness of such predictive models depends largely on the accuracy with which the involved parameters can be determined. Giacobbo et al. [16] have investigated the feasibility of using genetic algorithms for estimating the parameters of a groundwater contaminant transport model. Additionally, Qin et al. [17] have given a comprehensive review on recent developments, advancements, challenges, and barriers associated with simulation 
and optimization techniques in supporting process control of petroleum waste management and site remediation.

\section{Problem characterization}

Near Surface Repositories are an option for the permanent storage of radioactive waste produced by Nuclear Power Plants, such as PWR, based on International Atomic Energy Agency (IAEA) recommendations and international practice. Technetium-99 (Tc-99) is an important radionuclide selected among those present in intermediate level wastes [18-20] to be used in this paper.

The distribution coefficient is the ratio of the amount of substance sorbed on a unit mass of dry solid to the concentration of the substance in a solution in contact with the solid, assuming equilibrium conditions. The SI units are $\mathrm{m}^{3} / \mathrm{kg}$. Therefore, if the distribution coefficient is zero, the substance is not retained by the medium, which is, in this case, the geosphere [21].

The radionuclide Tc-99 was chosen to have its behaviour studied in this work because its distribution coefficient in the geosphere presents value equal to zero $[19,22]$. Since the host environment (geosphere) does not possess the property to retain the radionuclide, the engineering structure (size and arrangement of the repository) becomes the only barrier against the release of Tc-99 into the biosphere. The activity considered for the Tc-99 is $2.38 \times 10^{10} \mathrm{~Bq}$ in the year of the repository closing.

The repository is divided in 6 subsystems: A - top cover; $\mathrm{B}$ - upper layer; $\mathrm{C}-$ packages; D - base; E - repository walls, and F - geosphere. Release of contaminated water from the base is taken into account as well as from the side walls. Subsystems A and B are barriers against water infiltration while subsystems C, D, E, and F are barriers which delay the leaching of the radionuclide into the biosphere (see fig. 1).

The repository is viewed as a system whose components (barriers) work in a series-parallel configuration according to fig. 2, where the system failure occurs only when the barriers A and B and C and (D or E) and F fail. The time to failure of each barrier is considered a random variable with a specified probability density function.

The barriers operate sequentially so that only one component of the system is operating at a given time while the others remain in reserve. The components in reserve do not fail in this operation mode. The failure of a unit increases the level of stress of the unit following, thus increasing the latter's failure rate. The failures are dependent events.

Water infiltration models determine the infiltration of water into waste disposal units by following the precipitation of water at a site and its partitioning due to surface runoff, evaporation, and plant uptake/evapotranspiration. Percolation of water into the waste is then determined by describing the flow through the cover system, concrete vault, and other barrier components. Precise estimation of water flow is extremely complicated due to localized variability of material properties, transient nature of rainfall events, etc. Therefore, 


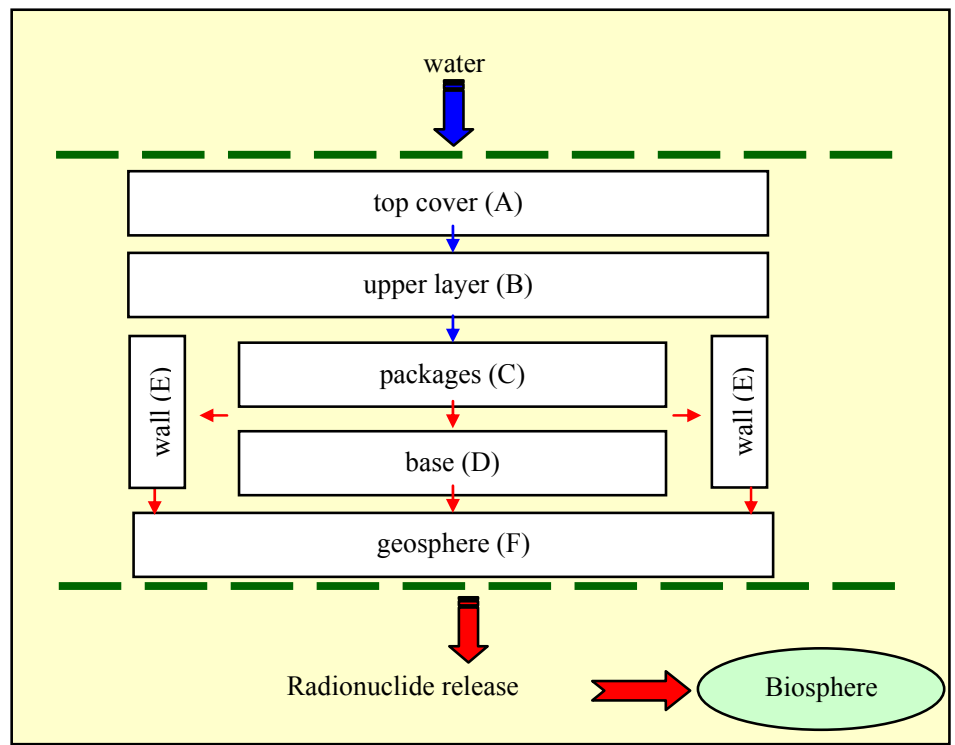

Figure 1: $\quad$ Schematic figure of the barriers model.

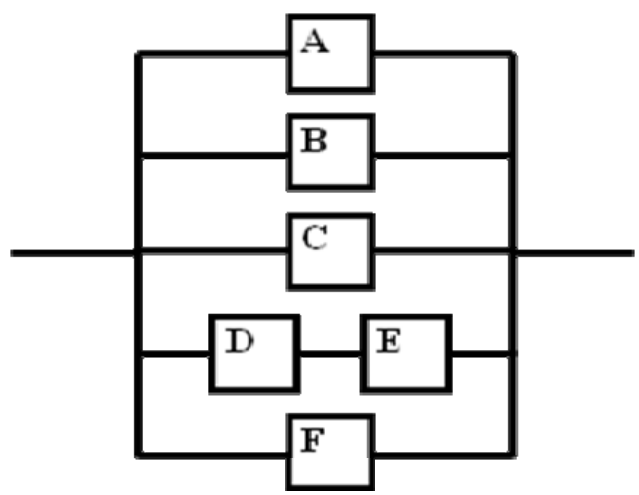

Figure 2: $\quad$ Series-parallel configuration of the repository barriers.

simplifications are made in performance assessment with the aim of obtaining a defensible estimate of water flow [23].

Infiltration predicted by the water infiltration models becomes important only after the failure of a concrete barrier in performance assessment. Before a failure, it is assumed that a concrete vault works as an effective barrier to minimize the contact of water with the waste. The time of failure of concrete vault is important, along with the time interval between the initiation and completion of concrete vault failure. The performance history of modern concrete is short $(-100$ years) relative to the required time frame of performance assessment, therefore, prediction of concrete service life is difficult [24]. 
A saturated porous medium is considered in order to calculate the superficial speed of the water referent to the total transversal draining area through the barriers. It is admitted that no infiltration will happen due to the active institutional control in the first 100 years $[18,20]$. Hence, the time the water takes to cross the concrete top cover will be added by 100 years. The water speed is obtained from the Darcy's equation for a porous saturated medium, except geosphere, eqns. (1) to (7).

$$
\begin{gathered}
v a_{1}(t)=k(t) \frac{\left(h+e_{A}\right)}{e_{A}}=k(t) \frac{H}{e_{A}} \\
v a_{2}(t)=k(t) \frac{\left(H+e_{B}\right)}{e_{B}} \\
v a_{3 v}(t)=k(t) \frac{\left(H+e_{B}+e_{C v}\right)}{e_{C v}} \\
v a_{3 h}(t)=k(t) \frac{\left(H+e_{B}+e_{C v}\right) / 2}{e_{C h}} \\
v a_{4}(t)=k(t) \frac{\left(H+e_{B}+e_{C v}+e_{D}\right)}{e_{D}} \\
v a_{5}(t)=k(t) \frac{\left(H+e_{B}+e_{C v}\right) / 2}{e_{E}} \\
v a_{6}=\frac{K_{g} \cdot i_{g}}{p_{g}}
\end{gathered}
$$

where,

$k(t)$ is the concrete hydraulic conductivity, given by eqn. (8);

$h$ is the water column height above the concrete top cover (subsystem A);

$H$ is the height corresponding to the thickness of the concrete top cover $\left(e_{A}\right)$ plus the height $h$;

$e_{B}$ is the thickness of the upper layer (subsystem B);

$e_{C v}$ is the thickness of the packages in the vertical direction (subsystem C);

$e_{C h}$ is the thickness of the packages in the horizontal direction (subsystem C);

$e_{D}$ is the thickness of the base (subsystem D);

$e_{E}$ is the thickness of the repository walls (subsystem E).

$$
k(t)=3 \cdot 10^{-7} t^{2}-10^{-4} t+8.10^{-7}
$$

The radionuclide speed equals the water speed divided by the retardation coefficient of the medium. Thus, the radionuclide Tc-99 speed is obtained by eqn. (9).

$$
v r(t)=\frac{v a(t)}{F r(t)}
$$


The radionuclide retardation coefficient can be determined by eqn. (10):

$$
F r(t)=1+\left(\frac{1-p(t)}{p(t)}\right) \rho(t) \cdot k d(t)
$$

The radionuclide retardation is the reduction in the rate of radionuclide movement through the soil due to the interaction (e.g. by sorption) with the immobile matrix. The retardation coefficient is a measure of capability of the porous media to impede the movement of a particular radionuclide been carried by fluid.

As Tc-99 presents distribution coefficient equal zero in geosphere, the substance is not retained, which implies in a retardation coefficient equal to 1 .

The equations for the porosity, and specific mass of the concrete and distribution coefficient $(k d(t))$ for radionuclides Tc-99 are respectively [22]:

$$
\begin{gathered}
p(t)=3 \cdot 10^{-7} t^{2}+5.10^{-4} t+0,15 \\
\rho(t)=-7.10^{-4} t^{2}-1.375 t+2500 \\
k d(t)=0,05 e^{-0,0046 t}
\end{gathered}
$$

The mean time to failure (MTTF) of each barrier equals the water average transit time (barriers A and B) or the radionuclide (barriers C, D, E, and F). The average transit time is the ratio between the water route of the radionuclide (barrier thickness) and its average speed.

The amount of activity released per year $(\mathrm{Bq} / \mathrm{y})$ is therefore the product between the probability density function, pdf $(1 / \mathrm{y})$, and the activity $(\mathrm{Bq})[1,25]$. The pdf related to the release of Tc- 99 caused by the barriers failure, due to rain water infiltration, is given by eqn. (14) [22]:

$$
f_{s i s}^{k}(t)=\frac{\left(\psi_{1}+\psi_{2}+\psi_{3}+\psi_{4}+\psi_{5}\right)}{\prod_{n=1}^{11} \Lambda_{n}}
$$

where,

$$
\begin{aligned}
& \psi_{1}=+\lambda_{A} \cdot \lambda_{B} \cdot \lambda_{C} \cdot \lambda_{F} \cdot e^{-\lambda_{F} \cdot t} \cdot \Lambda_{1} \cdot \Lambda_{2} \cdot \Lambda_{3} \cdot \Lambda_{5} \cdot \Lambda_{6} \cdot \Lambda_{8} \cdot\left(\Lambda_{10}\right)^{2} \\
& \psi_{2}=-\lambda_{A} \cdot \lambda_{B} \cdot \lambda_{C} \cdot\left(\lambda_{D}+\lambda_{E}\right) \cdot \lambda_{F} \cdot e^{-\left(\lambda_{D}+\lambda_{E}\right) t} \cdot \Lambda_{1} \cdot \Lambda_{2} \cdot \Lambda_{4} \cdot \Lambda_{5} \cdot \Lambda_{7} \cdot \Lambda_{9} \cdot \Lambda_{10} \\
& \psi_{3}=+\lambda_{A} \cdot \lambda_{B} \cdot \lambda_{C} \cdot \lambda_{F} \cdot e^{-\lambda_{C} \cdot t} \cdot \Lambda_{1} \cdot \Lambda_{3} \cdot \Lambda_{4} \cdot \Lambda_{6} \cdot \Lambda_{7} \cdot\left(\Lambda_{10}\right)^{2} \cdot \Lambda_{11} \\
& \psi_{4}=-\lambda_{A} \cdot \lambda_{B} \cdot \lambda_{C} \cdot \lambda_{F} \cdot e^{-\lambda_{B} \cdot t} \cdot \Lambda_{2} \cdot \Lambda_{3} \cdot \Lambda_{4} \cdot \Lambda_{8} \cdot \Lambda_{9} \cdot\left(\Lambda_{10}\right)^{2} \cdot \Lambda_{11} \\
& \psi_{5}=+\lambda_{A} \cdot \lambda_{B} \cdot \lambda_{C} \cdot \lambda_{F} \cdot e^{-\lambda_{A} \cdot t} \cdot \Lambda_{5} \cdot \Lambda_{6} \cdot \Lambda_{7} \cdot \Lambda_{8} \cdot \Lambda_{9} \cdot\left(\Lambda_{10}\right)^{2} \cdot \Lambda_{11}
\end{aligned}
$$




$$
\begin{aligned}
& \Lambda_{1}=\lambda_{A}-\lambda_{B} \quad \Lambda_{5}=\lambda_{B}-\lambda_{C} \\
& \begin{array}{l}
\Lambda_{2}=\lambda_{A}-\lambda_{C} \\
\Lambda_{3}=\lambda_{A}-\lambda_{D}-\lambda_{E}
\end{array}, \Lambda_{6}=\lambda_{B}-\lambda_{D}-\lambda_{E}, \begin{array}{l}
\Lambda_{8}=\lambda_{C}-\lambda_{D}-\lambda_{E} \\
\Lambda_{9}=\lambda_{C}-\lambda_{F}
\end{array}, \\
& \Lambda_{4}=\lambda_{A}-\lambda_{F} \quad \Lambda_{7}=\lambda_{B}-\lambda_{F} \\
& \Lambda_{10}=\lambda_{D}+\lambda_{E} \\
& \Lambda_{6}=\lambda_{D}+\lambda_{E}-\lambda_{F}
\end{aligned}
$$

and $\lambda_{A}, \lambda_{B}, \lambda_{C}, \lambda_{D}, \lambda_{E}, \lambda_{F}$ are the failure rates of the barriers $\mathrm{A}, \mathrm{B}, \mathrm{C}, \mathrm{D}, \mathrm{E}$ and $\mathrm{F}$, respectively.

\section{Genetic algorithm}

A genetic algorithm is employed to establish an optimized configuration for the structure of the repository, by determining the thicknesses of barriers and waste packages arrangement, taking into account the engineering and radiologic constraints. Such restrictions aim to keep the risks associated with the migration of radionuclides at a low level.

As starting point for optimization, the following set of geometric and structural parameters of the repository is used, as shown in reference [1] (reference repository): A - top cover ( $0.40 \mathrm{~m}$ high); B - upper layer $(0.32 \mathrm{~m}$ high); $\mathrm{C}$ - packages (1.20m x 4 high, $1.40 \mathrm{~m} \times 17$ depth and $1.40 \mathrm{~m} \times 51$ width); D - base $(0.80 \mathrm{~m}$ high); E - repository walls $(0.60 \mathrm{~m}$ thick); and $\mathrm{F}$ - geosphere ( $40 \mathrm{~m}$ thick). The number of packages is equal to $3,468\left(\mathrm{n}^{\mathrm{o}}\right.$ pack $\mathrm{proj}$ ) and the total volume of concrete used to build the repository is equal to $3,314.06 \mathrm{~m}^{3}$ ( $\left(\mathrm{Vol}_{\text {total,proj }}\right)$.

The variables considered in the optimization process are: (i) the thicknesses of top cover, upper layer, walls and base; (ii) the number of packages in a pile, determining the height; (iii) the number of packages in a row, determining the width. The depth is obtained from the height and width in order to give a total number of packages as close as possible to 3,468 . The crossover type was the PMX as proposed by Goldberg [26].

The fitness function $\left(\mathrm{F}_{\mathrm{obj}}\right)$ is given by eqn. (15) and the release rate (eqn. (14)) is used as a constraint for the optimization. The $\mathrm{F}_{\text {obj }}$ is used to minimize the total volume of concrete weighted by the reference volume $\left(\mathrm{Vol}_{\text {total,proj }}\right)$ also considering the ratio between the number of packages in the reference repository $\left(\mathrm{n}^{\mathrm{o}} \mathrm{pack}_{\mathrm{proj}}\right)$ and in the modelled repository ( $\mathrm{n}^{\mathrm{o}}$ pack).The constraint establishes that the time-dependent release rate cannot exceed the value of $9.8550 \times 10^{5}$ $\mathrm{Bq} /$ year at any time. This value corresponds to $90 \%$ of the maximum release rate associated with the project presented in reference [1], which is $1.0950 \times 10^{6}$ $\mathrm{Bq} /$ year. This involves a safety margin of $10 \%$. The minimum value used for the thicknesses of the wall, cover and base was $0.40 \mathrm{~m}$, and a minimum value of $0.10 \mathrm{~m}$ to the top layer. 


$$
F_{\text {obj }}=\frac{\text { Vol }_{\text {total }}}{V_{\text {ol }}}\left(1+\frac{n^{o} \text { pact }, \text { proj }_{\text {proj }}}{n^{o} \text { pack }}\right)
$$

where,

$\mathrm{Vol}_{\text {total }}$ is the concrete volume corresponding to the base, walls, top cover and upper layer for the modelled repository;

$\mathrm{Vol}_{\text {total,proj }}$ is the concrete volume corresponding to the base, walls, top cover and upper layer for according to the geometric and structural parameters presented in reference [22];

$\mathrm{n}^{\mathrm{o}}$ pack $_{\text {proj }}$ is the number of packages conditioned within the repository presented in reference [22];

$\mathrm{n}^{\mathrm{o}}$ pack is the number of packages conditioned within the modelled repository

\section{Results and discussion}

Although Tc-99 presents a distribution coefficient in concrete equal to 81,567, which is a high value, the value of the retardation coefficient in the geosphere is equal to 1 , that is, there is no retention of Tc in the geosphere. This fact implies a reduction of the MTTF and, consequently, an increase of the probability of repository failure for this radionuclide [27]. In this modelling, where the sorption is one of the main phenomena considered, it is observed that the probability of the repository failure is related directly with the retardation coefficient (Fr) of the radionuclide in the medium (concrete and geosphere). As the release rate of Tc is obtained from the probability of repository failure (cumulated density function to system failure), the increase in the probability influences the release of the radionuclide into the biosphere.

The optimization process was carried out through exhausting search using software based on genetic algorithms in order to minimize $\mathrm{F}_{\text {obj }}$ given by eqn. (15).

Considering the constraint for the release rate, the best solution obtained for $\mathrm{F}_{\mathrm{obj}}$ in order to minimize the volume of concrete was 1.1529 . The values of the modelled parameters, according to the best solution, are presented in Table 1.

The best solution establishes the same number of packages in a column defined in the project of the reference repository, that is, four packages in a pile. Therefore, the best distribution for the packages is a $28 \times 31$ arrangement, while the reference repository has a $17 \times 51$ arrangement.

As a result, the modelling shows that it is possible to consider near surface repository projects presenting lower thicknesses without loss of system reliability.

The research shows that, in this modelled case, the most important factor is the number of packages in a pile. The bigger the column, the greater the pressure on the inferior layers of the package set, where there are radionuclides susceptible to release. In addition, the height of this column determines the pressure exercised on the walls and base of the repository, simultaneously. 
Table 1: Values for the modelled parameters and reference repository (project).

\begin{tabular}{|c|c|c|}
\hline & Modelled & Project \\
\hline Top cover $(\mathrm{m})$ & 0.40 & 0.40 \\
\hline Upper layer $(\mathrm{m})$ & 0.10 & 0.32 \\
\hline $\mathrm{N}^{0}$ Packages & 3,472 & 3,468 \\
\hline height & 4 & 4 \\
\hline depth & 28 & 17 \\
\hline width & 31 & 51 \\
\hline Base $(\mathrm{m})$ & 0.40 & 0.80 \\
\hline Walls $(\mathrm{m})$ & 0.40 & 0.60 \\
\hline Volume $\left(\mathrm{m}^{3}\right)$ & $1,911,46$ & $3,314.06$ \\
\hline Release rate $(\mathrm{Bq} / \mathrm{a})$ & $8.2050 \times 10^{5}$ & $1.0950 \times 10^{6}$ \\
\hline
\end{tabular}

Some tests carried out in this work show that for certain limit values established for the release rate (constraint), the safety criteria can be reached by increasing the thicknesses of the barriers rather than reducing the number of packages per pile. However, this possibility occurs only in a few narrow ranges of release rate limits.

Performance assessment relies upon predictive mathematical/computer models to project the performance of a site for long periods of time. Due to the limited nature of the available data and models and the need to extrapolate the known database in time, uncertainties naturally arise. These include model and parameter uncertainties.

Our results demonstrate the behaviour of the selected radionuclide Tc-99 according to this modelling.

\section{Conclusion}

This work presented an approach to a model in order to establish an optimized configuration for the structure of repositories by determining the thicknesses of barriers and waste package arrangement, taking into account the engineering and radiological constraints.

The genetic modelling employed had embedded the function to minimize the total volume of concrete, weighted by the reference volume $\left(\mathrm{Vol}_{\text {total,proj }}\right)$, and considering the ratio between the number of packages according to the reference $\left(\mathrm{n}^{\mathrm{o}} \mathrm{pack}_{\mathrm{proj}}\right)$ and the number of packages by modelling ( $\mathrm{n}^{\mathrm{o}}$ pack). The results are fully satisfactory and compatible with the system studied, demonstrating the effectiveness of the proposed approach for this kind of problems.

The release rate is mainly sensitive to the number of packages per pile, more than to the thickness of the barriers. In order to obtain the lower concrete volume, the number of packages per pile must be defined to give the repository a structure as close as possible to a "cubic" geometry, always considering the constraint associated to the release rate limit. 
The modelling shows that it is possible to consider the construction of near surface repositories with lower thicknesses without loss of system reliability.

For radionuclides other than Tc-99, with release rates influenced by the geosphere, the thicknesses of barriers are probably not significant, as the geosphere has a much greater thickness. At this point, it is proposed that this study be extended to the other radionuclides existing in low and intermediate activity wastes which can be disposed in a near surface repository. For a more accurate study, it is suggested to consider the uncertainties in performance assessment.

\section{Acknowledgements}

The authors would like to thank all of the reviewers who provided valuable comments to this paper. The authors are very grateful to CTEx (Brazilian Army Technological Centre) and the Centre for Environmental Systems Analysis for supporting this work.

\section{References}

[1] Nair, R.N. \& Krishnamoorthy T.M., Probabilistic safety assessment model for near surface radioactive waste disposal facilities, Environmental Modelling \& Software 14, 447-460, 1999

[2] Aguiar, L. A.; Alves, A. S.; Passos, E. M; Frutuoso and Melo, P.F. Probabilidade de liberação líquida de radionuclídeos de um Repositório Próximo à Superfície. Proceedings of ENAN / International Nuclear Atlantic Conference (INAC 2005). Santos - SP, 2005.

[3] IAEA, Scientific and technical basis for the near surface disposal of low and intermediate level waste, Technical Reports Series No. 412, Vienna, 2002.

[4] Bertozzi, G., D'alessandro, M., Girardi, F., and Vanassi, M. Safety assessment of radioactive disposal into geological formation. EUR-5901, Commission of the European Community, Luxemburg, (1978).

[5] Pritzker, A. and Gassmann, J. Application of simplified reliability methods for risk assessment of nuclear waste repository. Nuclear Technology 48:289 (1980).

[6] Garrick, John B., The use of risk assessment to evaluate waste disposal facilities in the United States of America, Safety Science 40, 135-151, 2002.

[7] Malbrain, C. M. Risk assessment and the regulation of high level waste repository. Doctoral dissertation, Massachusetts Institute of Technology, Cambridge (1984).

[8] Kim, P. O., Cho, W. J., and Chang, S. H. Probabilistic safety assessment of low level waste disposal system. Radioactive Waste Management and the Nuclear Fuel Cycle 10:253 (1988). 
[9] Han, K. W., Cho, W. J., Kang, C. H., and Kim, C. H. Genetic safety assessment for LLW repository. Proceedings of the 1991 Joint International Waste Management Conference 1:107 (1991).

[10] Little, R.H. \& Penfold, J.S.S. Preliminary Safety Assessment of Concepts for a Permanent Waste Repository at the Western Waste Management Facility Bruce Site: Summary Report, Quintessa Limited, Ontario - Canada, march 2003.

[11] Ene, Daniela, Test Case of the Long Term Preliminary Performance Assessment for the L\&IL Radioactive Waste Repository Baita Bihor, Romania, ICRS-10/RPS Madeira, 2004

[12] Chesnut, D. A., Demands placed on waste package performance testing and modeling by some general results of reliability analysis, Nuclear Technology, v104, Nov. 1993

[13] Ananda, M. M. A., Reliability modeling of engineered barrier systems for nuclear waste: a conditional approach. Microelectronics Reliability, vol 34, $n$ 7, 1994.

[14] Ananda, Malwane M. A. \& Singh, Ashok K., Performance assessment of multiple engineered barrier systems. Applied Mathematics and Computation, 102, 1999.

[15] Aguiar, L.A.; Alves, A. S.; Passos, E. M; Frutuoso e Melo, P.F. Confiabilidade de embalados de rejeitos radioativos em repositórios próximos à superfície. Proceedings of ENAN / International Nuclear Atlantic Conference (INAC 2005). Santos - SP, 2005a.

[16] F. Giacobbo, M. Marseguerra, E. Zio. Solving the inverse problem of parameter estimation by genetic algorithms: the case of a groundwater contaminant transport model. Annals of Nuclear Energy 29 (2002) 967-981

[17] X.S. Qin, G.H. Huang, L. He. Simulation and optimization technologies for petroleum waste management and remediation process control. Journal of Environmental Management 90 (2009) 54-76

[18] IAEA, Safety assessment methodologies for near surface disposal facilities, results of a coordinated research project. Volume $1-$ Review and enhancement of safety assessment approaches and tools, Vienna, 2004a.

[19] Vieno, T. \& Nordman, H., VLJ Repository safety analysis. Report TVO 1/98, Finland, July 1998.

[20] IAEA, Safety assessment methodologies for near surface disposal facilities, results of a coordinated research project. Volume 2 - test cases, Vienna, $2004 b$.

[21] IAEA, Radioactive waste management glossary, IAEA, Vienna, 2003.

[22] Aguiar, L.A. Avaliação de risco de um repositório próximo à superfície na fase pós-fechamento em cenário de liberação de radionuclídeos por infiltração de água. D.Sc. Thesis. COPPE/UFRJ. Rio de Janeiro, 2006.

[23] Man-Sung Yim; Scott A. Simonson. Performance Assessment Models for Low Level Radioactive Waste Disposal Facilities: A Review. Progress in Nuclear Energy, Vol. 36, No. 1, pp. 1-38, 2000.

[24] J. C. Walton, L. E. Plansky, and R. W. Smith. Models for Estimation of Service Life of Concrete Barriers in Low-Level Radioactive Waste 
246 Waste Management and the Environment V

Disposal. NUREG/CR-5542, EGG-2597, U. S. Nuclear Regulatory Commission, 1990.

[25] Cho, Won-Jin; Chang, Soon-Heung \& Park, Hum-Hwee, Uncertainty analysis of safety assessment for high-level radioactive waste repository. Waste Management, v 12, 1992

[26] Goldberg, D.E. 1989. Genetic algorithms in search, optimization, and machine learning. Addison-Wesley Professional, USA.

[27] Aguiar, L.A.; Frutuoso e Melo, P.F; Alvim, A.C.M. Estimation of mean time to failure of a near surface radioactive waste repository for PWR power stations. Proceedings of ENAN / International Nuclear Atlantic Conference (INAC 2007). Santos - SP, 2007. 